\title{
A Task-oriented Conceptual Design Method for New Types of Equipment
}

\author{
Bin Liu, Tonggang Yu, Baojun Wu, Kaiquan Xiang \\ Shijiazhuang Campus of Army Engineering University, Shijiazhuang 050003, China \\ robin8402@163.com
}

Keywords: conceptual design, task-oriented, function, behavior, structure.

Abstract: In the new equipment design process, the conceptual design phase is at the beginning of all designs, but it is also the most important phase. Therefore, researching and implementing the functions of new equipment is the core issue of its conceptual design. It has very important practical significance to improve the innovation and efficiency of new equipment design, reduce equipment cost and improve competitiveness. By referring to relevant research results at home and abroad, this paper combines the function, behavior and structure features of new equipment, and combines the task-oriented new equipment design process, proposes a FBS method, and gives specific implementation steps and applications. Examples can provide a new research approach for new equipment concept design.

\section{Introduction}

With the development of science and technology, new types of equipment are appearing more and more in our lives, and their effective design has received more and more attention. Relevant research shows that although the actual investment in the design stage only accounts for a small proportion of the total cost of equipment production, the impact on the subsequent links is extremely far-reaching, which determines $70 \%-80 \%$ of the total equipment cost. In the whole design process, conceptual design is the most important part, which is the most creative stage in the design process, and also the main means to improve equipment quality and reduce risks [1]. If the conceptual design of the new equipment is flawed, the latter design will not be able to make up even if it is perfect. Therefore, we should pay special attention to the conceptual design of new equipment.

\section{Conceptual design}

Conceptual design is an important stage of product design. To clarify the connotation of conceptual design, we must first understand the concept of product design, but different scholars have different divisions of product design. For example, Pahl and Beitz divide product design into Defining the four stages of task, concept design, technical design and construction design. Although the above scholars have different divisions in the product design stage, by analyzing these points, 
they can be found that they have a common feature - they all think that product design is a simple to complex, macro to micro development process, and ultimately must be formed a product design.

According to the above analysis, this paper gives the definition of conceptual design: conceptual design is to abstract the task, abstract the functional structure, seek appropriate action principle and combination, determine the basic solution path, and get the solution. The process of the program.

Conceptual design is the stage in which the designer's thinking has a preliminary design plan but has not yet formed a specific plan. The purpose is to help the designer to turn the ideas in his mind into concrete plans as soon as possible. Conceptual design has the typical characteristics of innovation, diversity, hierarchy and dynamics. For conceptual designers, a clear understanding of these characteristics is conducive to improving the level of conceptual design and making the new equipment design more targeted.

\section{Task-oriented conceptual design method}

The new equipment concept design process is a complex and uncertain design reasoning process, which can also be called a conceptual design process model. In the end, which problems are selected to solve, this is the concept designer. Need to focus on. This paper believes that in the process of designing new equipment concept, we must first consider the task of new equipment. On the basis of task decomposition, we should focus on the three elements of equipment function, behavior and structure to form a "function-behavior-structure" model., that is, the FBS model, in which the function expresses the purpose of conceptual design, that is, "what to do", and the behavior represents the method that the designer uses to infer the structure, that is, "how to do it", which can also be interpreted as a principle, and the structure implies what components of the design are made up, the properties of these components, and the relationship between the components, that is, "what it is like.” Behavior acts as a bridge between function and structure.

In this case, there are mainly two types of mapping: F-B (function-behavior) mapping and B-S (behavior-structure) mapping. In order to better understand the two types of mapping, the three processes of function analysis, behavior analysis and structure analysis need to be elaborated.

\subsection{Function Analysis}

The result of function analysis is the generation of a collection of functional components, the functional domain. The function is to abstractly describe the causal relationship between the equipment input object and the output object. In the early stages of function analysis, the overall function can be thought of as a black box, regardless of its specific structure, considering only the relationship between functions and external objects. The matter may be a liquid, a gas or various forms of solids, such as raw materials, components, finished products, etc.; the energy may be an energy flow in the form of mechanical energy, thermal energy, electrical energy, etc.; the information may be various input commands, data, images, and so on.

For specific new equipment, function refers to the effectiveness, use and function of the equipment. For example, the function of the transport tool is to transport personnel and materials; the function of the electric motor is to convert electrical energy into mechanical energy; the function of the reducer is to transmit torque and change the rotational speed; the function of the machine tool is to turn the blank into parts and so on. The overall function of the equipment is usually composed of many sub-functions or basic function elements, which are combined in an orderly logical relationship.

In the design of new type equipment concepts, the total task is generally more complicated, and the total function is also complicated, which is not conducive to the simplification and analysis of the problem. In order to further realize the mapping of functions, behaviors, and structures, function 
analysis can be performed by decomposing complex functions layer by layer according to system hierarchy. In order to clearly describe the relationship between complex functions and basic functions, we use the concept of sub-functions. A sub-function is actually a local complex function that can continue to be broken down into multiple sub-function or basic functions. How many sub-functions a complex function needs to be decomposed into, that is, the "granularity" of functional decomposition, often follows two principles: the principle of consistency and the principle of independence. Among them, the principle of consistency means that the sub-function after decomposition must be compatible with the original complex function. The principle of independence means that the sub-functions and basic functions formed by the decomposition of complex functions should be independent units with clear meaning, and the functions are loose. Coupling, less dependencies. Following the above principles in the functional decomposition process will facilitate the reuse of functions.

In the conceptual design process, there is a mapping relationship between functions and action objects (input objects and output objects), and this mapping relationship is an essential feature shared by such functions. Therefore, functions can be expressed in the following forms. That is, "noun + verb + noun”, where the verb is the verb meaning, and the noun is the input and output stream.

\subsection{Behavior analysis}

Behavior analysis describes how to implement a function that should be "if done", embodied in a series of behaviors, or as a principle. The new type equipment realizes its function through the process of interaction with the working object and the working environment. In the course of its work, some components generate energy, some components convert energy, and some components consume energy. Through the energy transfer, conversion or consumption between components, it will exhibit certain behavioral characteristics. Behavioral characteristics can be divided into static behavior and dynamic behavior. Static behavior refers to the behavior that a system or component cannot be perceived by human beings, generally characterized by material properties such as stress and support. Dynamic behavior means that a system or component can be perceived by a person to change state over time, such as spring force, torque.

Although behavior has many different attributes, not every attribute has a positive, direct or indirect effect on the function implementation of the equipment. Therefore, designers often focus on the main attributes in many attributes. For example, the behavioral attributes considered by tanks when climbing a hill are mainly "how much kinetic energy is generated, allowing them to pass quickly." The main behavioral attributes considered by the cutting machine when cutting ice cubes are "cutting speed and cutting force", etc. Therefore, in the design of new type equipment concepts, we must pay attention to the main behavioral attributes and seize the main contradictions.

The implementation of behavior is mainly embodied in a series of physical principles. Therefore, when describing behaviors, it can be expressed by corresponding physical principles. For example, there are several kinds of physical principles such as levers and pulleys for force amplification.

\subsection{Structure analysis}

The result of structure analysis is the formation of a series of structures that are the carriers required for the conceptual design and the carrier for the intended function. The Structure design in the conceptual design equipment is based on the principle design to determine the feature structure, shape and size of the entire concept equipment. In this process, the details of each component are not involved, but the functional requirements are mainly considered. How to be satisfied at the level of geometry and structure. The structure mainly refers to the mechanical structure in the design catalog, 
such as gear mechanism, linkage mechanism, cam mechanism and the like.

In order to realize the structure in the design concept of the new type equipment, the structure modeling of the equipment must be carried out. The purpose of structure modeling is to create an abstract description of the physical structure of the equipment that produces behavior and implements functionality. The structure model is the basis of detailed engineering design, and its basic composition is the basic unit. The basic unit is a structure that produces the behavior represented by the behavior unit. It consists of points, lines, and surfaces, which is equivalent to a simplified diagram of the parts. The basic structure units of different fields are different. The structure of the electromechanical system, such as the linkage mechanism and the screw mechanism. The structure of the mechanical system such as lever, friction, etc.

The attributes of a structural unit refer to structure features. The attribute definitions may include names, functions implemented, etc. The description of the attributes may be supported by many CAD tools, such as STEP, using standardized neutral forms. Describe the geometric characteristics of the components, which are not highlighted here.

Since the new type equipment concept design stage often uses sketching methods to inspire ideas and represent shapes, the topological relationship is the premise of entering the detailed design in the late stage of the conceptual design stage. The geometric information of the principle structure model is refined and improved in the detailed design stage. To express the topological relationship, three topological methods are needed: spatial relationship, capturing the position of the component space; connection relationship, capturing the degree of freedom of the physical relationship; and descriptive symbols, capturing the essence of the transmission of energy in the topological essence.

\section{Case Analysis}

This paper takes the conceptual design of new mechanical icebreaking equipment as an example to illustrate the application of FBS model. The task of mechanical ice-breaking equipment is to break down river ice blast by means of mechanical movement. It can be easily divided into two parts: equipment carrying platform and ice-breaking device. According to the supporting foundation on which the platform is supported, it is mainly divided into the following five types of equipment: shore-based equipment. , surface equipment, underwater equipment, ice equipment and hybrid equipment, the following mainly describes the conceptual design process of shore-based equipment.

\subsection{Function analysis}

The main function of the shore-based equipment (use function) is "breaking ice on the shore", that is, breaking the ice on the river bank, decomposing this function, forming a first-order sub-function, namely "shore maneuver", "equipment fixed", "breaking point choose and "vibration hammer operation" continue to perform second-order decomposition of functions. The decomposition function of "equipment maneuvering” is "adjustment speed", "temporary stop", "change direction" and so on.

\subsection{Behavior analysis}

The main behavior of using shore-based equipment to implement icebreaking is to fix the load-bearing equipment on the outside of the water-shore boundary line and select suitable power equipment (usually with a vibrating hammer) to make the ice surface rupture by using the crushing function of the power equipment. 


\subsection{Structure analysis}

Depending on the position of the mechanical platform, a full shore test and an ice shore test can be performed. Among them, the complete shore-based test is a vibration breaking test in which the power equipment and the supporting equipment of the vibrating hammer are placed outside the boundary line of the ice bank. Usually, the fulcrum of the crane is not allowed on the ice surface; the ice foundation test is the vibration hammer. The power equipment and the carrying equipment are placed in a vibration breaking test between the water boundary line and the ice bank boundary line, and the equipment can be operated on the ice surface.

Through the above analysis, we believe that the advantages of shore-based equipment is that the load-bearing equipment has better safety, simple structure, lower cost and easy to be applied on a large scale. The disadvantage is that the range of ice-breaking is limited, and only the ice surface on the shore can be broken. The ice surface in the middle is not effective, and when the ice-breaking mechanism breaks the ice, its crushing power is its own gravity, and the platform only provides upward pulling force, so the ice-breaking efficiency is low.

\section{Conclusions}

The task-oriented new type equipment concept design method is a wide-ranging research topic, and it contains a lot of content. Based on the process of conceptual design, this paper takes the functional, behavioral and structure features of the new type equipment as the object of investigation, and proposes a new type equipment conceptual design model based on FBS. The model is verified and the results have certain validity and feasibility. The follow-up needs to study the concept design knowledge base and the concept design evaluation system to further improve the innovation and efficiency of the new type equipment concept design, and bring the level of new type equipment concept design to a new level.

\section{References}

[1] Y.C. Liu,J.M. Wu, B. Liu, et al, Research on FBS Model of New Equipment Conceptual Design, Journal of Ordnance Engineering College, 26 (2014) 5-8.

[2] Feng Er-Qiang, Zhen Jun,Lan Xin-Sheng. Study on software and hardware integrated reliability evaluation method. Application of Electronic Technique,2015,41(3):20-23

[3] Li Jing, Wang Gang, Liu Xiao-Guang,et al. Review of reliablity prediction for storage system. Journal of Frontiers of Computer Science and Technology,2017,11(3):341-354.

[4] Li He-Tian, Liu Yun, He De-Quan. Risk Assessment Model of Reliability for Information System. JOURNAL OF BEIJING JIAOTONG UNIVERSITY,2005,29(2)

[5] Hu Zhen-Qiang, Shang Ying-Jun. Analysis on reliability factors of software cycle. Computer and Network, 2017, 10(4):70-75. 